\title{
CARACTERÍSTICAS MORFOFUNCIONAIS DO TRÂNSITO OROFARÍNGEO NA BULIMIA: REVISÃO DE LITERATURA
}

\section{Morphofunctional characteristics of the oropharyngeal tract in bulimia: review of literature}

\author{
Catarina Matos Brito Santos (1), Vanessa de Oliveira Cansanção (2), \\ Leandro de Araújo Pernambuco ${ }^{(3)}$, Hilton Justino da Silva ${ }^{(4)}$
}

\begin{abstract}
RESUMO
Tema: deglutição e bulimia. Objetivo: apresentar e discutir os achados científicos descritos na literatura quanto às características orofaríngeas relacionadas à deglutição em portadores de bulimia nervosa do tipo purgativa. Conclusão: a bulimia nervosa acarreta uma série de alterações em estruturas e funções que compõem o trânsito orofaríngeo, como erosão dentária, hipersensibilidade, enfraquecimento e fratura dos dentes, problemas de oclusão, cáries, doenças periodontais, dessensibilização intra-oral, hipogeusia, úlceras, granulomas, queilite angular, hipertrofia das glândulas parótidas, tosse e odinofagia. Existe um predomínio na literatura científica de relatos sobre alterações morfológicas em detrimento das funcionais. Poucos relatos abordaram diretamente a relação entre a bulimia e deglutição, apenas mencionando superficialmente as possibilidades de desencadeamento da disfagia orofaríngea.
\end{abstract}

DESCRITORES: Transtornos da Alimentação; Bulimia; Deglutição

\section{INTRODUÇÃO}

Os transtornos alimentares (TA) são quadros psiquiátricos ${ }^{1}$ caracterizados por graves alterações do comportamento alimentar ${ }^{1-4}$ e constituem um dos problemas de saúde de maior crescimento na população jovem ${ }^{5}$. Essa população é a mais atingida ${ }^{4,6,7}$ porque é neste período que ocorrem

(1) Fonoaudióloga; Especialização em Motricidade Orofacial: Enfoque em Disfagia pela Faculdade Integrada do Recife.

(2) Fonoaudióloga; Especialização em Motricidade Orofacial Enfoque em Disfagia pela Faculdade Integrada do Recife.

(3) Fonoaudiólogo do Hospital de Câncer de Pernambuco, HCP, Recife, PE; Centro de Reabilitação e Fisioterapia Distrito I, Jaboatão dos Guararapes, PE; Centro de Fonoaudiologia de Pernambuco, CEFOPE, Recife, PE; Especialização em Motricidade Orofacial Enfoque em Disfagia pela Faculdade Integrada do Recife; Mestrando em Ciências da Saúde na Universidade Federal de Pernambuco.

(4) Fonoaudiólogo; Professor Adjunto I do curso de Fonoaudiologia da Universidade Federal de Pernambuco, UFPE, Recife, PE; Vice-coordenador da Pós-graduação em Patologia da Universidade Federal de Pernambuco, UFPE, Recife, PE; Doutor em Nutrição pela Universidade Federal de Pernambuco.

Conflito de interesses: inexistente grandes transformações físicas e emocionais em um curto espaço de tempo e quando os conceitos ainda estão sendo formados ${ }^{7}$. Ocorrem predominantemente em mulheres ${ }^{2,4,6}$ de todas as classes sociais, em faixa etária cada vez mais ampla (1321, média 17 anos) ${ }^{2}$, podendo originar prejuízos biológicos e psicológicos ${ }^{4}$, afetando a saúde física e emocional do paciente e prejudicando seu desempenho social e profissional ${ }^{4,8}$.

As principais variações dos TA são a anorexia nervosa (AN) e bulimia nervosa (BN). Indivíduos com AN e BN são caracterizados pelo perfeccionismo, compulsividade obsessiva e alterações de humor frequentes ${ }^{9}$. Por apresentarem significativos graus de morbidade e mortalidade, os transtornos alimentares, especialmente a bulimia, vem recebendo grande atenção dos profissionais da área da saúde.

O termo bulimia deriva do grego boulimos, que por sua vez, deriva da fusão de bous (boi) e limos (fome), literalmente "fome de boi" ${ }^{10}$. A BN consiste em repetições de episódios de compulsão alimentar, nos quais a pessoa ingere grandes quantidades de alimentos ${ }^{11}$ com pouco ou nenhum prazer ${ }^{12}$ de maneira muito rápida e com a sensação de perda 
de controle - os chamados episódios bulímicos ${ }^{4,6}$. A BN surge após um período de restrição alimentar, que pode ou não estar associado à perda de peso. A compulsão alimentar é seguida por vários tipos de compensações (vômito auto-induzido, exercícios físicos em excesso, abuso de laxativos/ diuréticos ou enemas) devido à ingestão excessiva de comida ${ }^{9,13}$. Considerando os tipos de compensações, a bulimia pode ser classificada em dois subtipos: purgativa e não purgativa. A purgativa é caracterizada pelo vômito auto-induzido ou o uso de laxativos/diuréticos para induzir perda de peso e contornar as sequelas da compulsão alimentar. Já a não purgativa é caracterizada por excesso de exercícios físicos. Variantes que não se encaixam bem nessa classificação incluem: pílulas de dieta ou hormônio tireoidiano, mastigar e jogar fora a comida, e em diabéticos, abusar de doses de insulina para induzir diurese osmótica e perda de peso ${ }^{14}$.

Poucos estudos examinaram os efeitos que o comportamento do indivíduo bulímico pode causar nas estruturas e funções orofaríngeas, particularmente a deglutição. Tal fato causa perplexidade, considerando que a deglutição e o vômito fisiologicamente utilizam as mesmas estruturas anatômicas, em sentidos opostos ${ }^{15}$. O envolvimento das mesmas estruturas anatômicas em ambas as funções e a repetição patológica dos episódios de vômito podem danificar o controle sensório motor para deglutição pela estimulação anormal do sistema e submeter a boca e faringe ao conteúdo do estômago ${ }^{15}$. Por essas razões, constatou-se a necessidade de traçar um panorama da literatura existente e, a partir dela, estimular o aprofundamento em estudos e pesquisas referentes a este transtorno alimentar.

Este artigo de revisão de literatura tem por objetivo apresentar e discutir os achados científicos quanto às características orofaríngeas relacionadas à deglutição em portadores de bulimia nervosa do tipo purgativa.

\section{MÉTODOS}

Trata-se de um estudo do tipo revisão de literatura. O levantamento bibliográfico foi realizado a partir de busca nas bases de dados MEDLINE e LILACS, vinculadas à biblioteca virtual BIREME (http://bireme.br) e na base Periódicos CAPES. Utilizaram-se os seguintes descritores, extraídos dos Descritores em Ciências da Saúde (DeCS) e cruzados da seguinte forma: bulimia (B), B AND deglutição. Além disso, foi incluído na busca o termo livre disfagia, dada sua importância dentro da proposta deste trabalho. Foram considerados os artigos originais e de revisão de literatura, publicados no período de 1997 a junho de 2008, nas línguas inglesa, espanhola e portuguesa, tendo sido excluídos trabalhos nos demais idiomas.

Foram selecionados 37 artigos para composição deste trabalho. Considerou-se para seleção, a presença de correlação, no título ou no resumo, entre bulimia, as estruturas que compõem o trato orofaríngeo e a função deglutição. A partir da análise destes trabalhos, foram identificados outros 9 artigos, sendo estes considerados relevantes, dada suas frequentes citações. Desta forma, no total, foram incluídos 46 artigos. A pesquisa ainda teve suporte de informações coletadas no site da National Eating Disorders Association.

A extração dos dados foi realizada por três revisores que consideraram as seguintes informações: autores, ano de publicação, local onde foi realizado o estudo, ano de publicação, faixa etária da população pesquisada, metodologia, definições de bulimia, epidemiologia, características morfológicas das estruturas do trato orofaríngeo, características funcionais do trato orofaríngeo, menção explícita à deglutição ou disfagia.

\section{REVISÃO DA LITERATURA}

\section{Bulimia}

O primeiro estudo que descreveu a bulimia foi o de Russel em 1979, no qual ele identificou os sujeitos baseado em dois critérios: episódios de compulsão seguidos de vômitos auto-induzidos ou purgantes e o medo de ficar gordo. Ele também descreveu os métodos que os pacientes usavam para prevenir o ganho de peso, bem como destacou o sinal de Russell, que consiste em uma calosidade ou escoriação no dorso da mão devido à introdução dos dedos na boca para provocar o vômito ${ }^{16}$.

A BN atinge de $1 \%$ a $2 \%$ da população, com maior prevalência no sexo feminino, em uma proporção de 9:1 em relação ao sexo masculino. $O$ início dos sintomas ocorre nos últimos anos da adolescência ou até os 40 anos, estando a média de início por volta dos 20 anos de idade ${ }^{4,12,17}$. Geralmente o perfil destes pacientes é composto por adolescentes do sexo feminino, raça branca e alto nível social, econômico e cultural ${ }^{4,12,14,17}$. Além disso, cerca de 25 a $30 \%$ dos bulímicos tem história prévia de anorexia ${ }^{9}$.

Acredita-se que a BN tenha origem multifatorial já que as causas estão associadas tanto a um nível neurobiológico como psicossocial ${ }^{18}$.

Está crescendo o consenso de que as vulnerabilidades neurobiológicas têm uma contribuição substancial na patogênese da BN. Evidências consideráveis sugerem que alterações na função serotoninérgica no cérebro $(5-\mathrm{HT})$ contribuem para 
modificações na regulação do apetite, humor e o controle dos impulsos na $\mathrm{BN}{ }^{9}$. A serotonina é um neurotransmissor e vasoconstrictor que estimula 0 músculo liso e diminui a secreção gástrica ${ }^{8}$. Também já foi relatada uma alteração nos níveis de colecistoquinina, o que sugere um desequilíbrio do mecanismo de saciedade. Talvez este fenômeno explique o porquê de pacientes com bulimia nervosa não se sentirem saciados após episódios de ingestão compulsiva de alimentos ${ }^{8,19}$. Ainda há um ponto de vista genético: estudos demonstraram que se a mãe ou irmã de uma mulher sofre de anorexia, seu risco de desenvolver a doença é 12 vezes maior e é 4 vezes maior para bulimia, considerando os aspectos psicossociais envolvidos ${ }^{14}$.

Do ponto de vista psiquiátrico, a bulimia é caracterizada como uma resposta inadequada ou destrutiva às relações interpessoais e está relacionada à depressão, baixa auto-estima, intolerância a frustrações, etc ${ }^{19,20}$. Socialmente, a bulimia está associada à busca da magreza como padrão de beleza, medo de engordar, exibição de corpos magros nos meios de comunicação etc ${ }^{20}$. A bulimia é, portanto, o resultado de múltiplas causas e da participação de diversos processos que, apesar de resultar nas mesmas manifestações, obedecem a causas diferentes, e dependendo do caso pode apresentar uma, várias ou todas as características supracitadas.

O diagnóstico da BN é feito segundo os critérios estabelecidos pela American Psychiatric
Association, através do Diagnostic and Statistical Manual of Mental Disorders, cuja última versão, o DSM IV, foi publicada em 1994 e pelo CID 10 (Classificação Internacional de Doenças) segundo a OMS (Organização Mundial de Saúde) (1992) ${ }^{10,21}$, conforme referidos na Tabela 1.

Estes pacientes escolhem os horários de refeições de modo que fiquem sozinhos para comerem compulsivamente; comem em recipientes grandes como panelas e tigelas, acompanhando as refeições com grandes volumes de líquidos para facilitar ou promover o vômito e ingerem rapidamente grandes quantidades de alimentos (8000 a 10000 $\mathrm{Kcal} / \mathrm{h})^{21}$.

Para neutralizar os episódios recorrentes de hiperfagia, esses indivíduos utilizam métodos compensatórios, com o intuito de prevenir o ganho de peso ${ }^{6,19,22,23}$. Pacientes bulímicos geralmente estão cerca de 3 a $5 \mathrm{Kg}$ abaixo do peso ${ }^{13}$. O método compensatório mais utilizado é a regurgitação auto-induzida ( $80 \%$ a $90 \%$ dos casos) ${ }^{19}$. A indução pode ser feita através de estimulação faríngea com os dedos, com a utilização de xarope de ipeca incluindo a ingestão crônica de aspirina ou podem ser espontâneas, basta ter aprendido, sem meio externo algum, a auto-provocá-los, por exemplo, simplesmente ao agachar-se ${ }^{10}$. Também podem fazer uso de medicamentos (diuréticos, laxantes, inibidores de apetite, hormônios tireoidianos), dietas restritivas, exercícios físicos em exagero (podendo causar danos aos ligamentos e músculos) e abuso

Tabela 1 - Critérios diagnósticos para bulimia nervosa - DSM IV (Diagnostic and Statistical Manual of Mental Disorders, American Psychiatric Association, 1994) ${ }^{10,12}$

\section{Critérios diagnósticos para bulimia nervosa}

a. Presença de episódios recorrentes de compulsão alimentar que pode se caracterizar por:

1. Ingesta de alimentos em um curto espaço de tempo (por exemplo, em um período de duas horas) e em quantidade superior a que a maioria das pessoas ingeriria em um período similar e nas mesmas circunstâncias.

2. Sensação de perda de controle alimentar durante o episódio.

b. Condutas compensatórias inapropriadas, de maneira repetida, com a finalidade de prevenir o ganho de peso, como a indução do vômito; uso excessivo de laxantes, diuréticos, enemas e outras drogas, jejum ou exercícios excessivos.

c. A compulsão alimentar e comportamentos compensatórios ocorrem duas vezes por semana, por, pelo menos, três meses.

d. Preocupação excessiva com a forma corporal e peso.

e. O distúrbio não ocorre durante os episódios de anorexia nervosa.

\section{Subtipos:}

Tipo purgativo: durante o episódio de bulimia nervosa, o indivíduo provoca regularmente o vômito fazendo uso de laxantes, diuréticos ou enemas em excesso.

Tipo não purgativo: durante o episódio de bulimia nervosa, o indivíduo faz uso de outras condutas compensatórias inapropriadas, como exercício em excesso e jejum prolongado. 
de cafeína ou uso de cocaína 4,6,10,17,19,22,24,25. Outros estudos apontam que este tipo de conduta bulímica também se observa em pacientes com diabetes mellitus, malária, e entre meninas que vivem longe das famílias em internatos ${ }^{8}$.

Os pacientes com bulimia apresentam sinais e sintomas de ordem geral que incluem metabolismo basal baixo, temperatura corporal baixa, pressão sanguínea baixa, pulso rápido, hipotonia muscular e risco de colapso circulatório. Em alguns casos podem ocorrer manifestações de depressão, retração social, irritabilidade, insônia, perda do interesse sexual e até mesmo amenorreia em determinadas mulheres ${ }^{26}$. Indivíduos com BN tendem a ser mais perfeccionistas, obsessivo-compulsivos e tensos. São mais impulsivos e buscam sensações estimulantes ${ }^{9}$. A BN não está associada a um aumento patológico do apetite. Os bulímicos têm uma tendência a restringir a dieta, um extremo medo de ganhar peso e casualmente tem uma visão distorcida de sua imagem corporal $^{9}$.

\section{Cavidade oral}

$\mathrm{Na}$ bulimia nervosa, as primeiras manifestações ocorrem através de alterações orais e maxilofaciais ${ }^{27}$, que podem se manifestar aproximadamente seis meses depois de uma pessoa consistentemente se submeter a comportamentos alimentares como o vômito ${ }^{28}$. Por ser um comportamento estranho à normalidade, acaba por facilitar o diagnóstico preciso da doença.

Por sua relação direta com essa região, o cirurgião-dentista pode ser o primeiro profissional de saúde a suspeitar da BN devido aos sinais e sintomas de erosão dental resultantes de um ambiente cronicamente ácido na região oral ${ }^{12}$, sendo este 0 sintoma mais relatado ${ }^{15}$.

A erosão dental é um processo de descalcificação que se manifesta lentamente ${ }^{19}$. Este tipo de erosão é facilmente diferenciado dos demais devido ao desgaste típico das faces palatal e bucal dos elementos anteriores da arcada dentária superior ${ }^{15}$. Ela não se apresenta a menos que a regurgitação tenha sido presente em, pelo menos, dois anos consecutivos. Portanto, o grau de erosão pode ser diferente em grau e velocidade de progressão, que, por sua vez, é dificultada por questões tais como a capacidade tampão salivar (buffer), $\mathrm{pH}$ salivar, amplitude fluxo salivar, composição e grau de calcificação superfície dental, bruxismo, tipo de dieta ácida (frutas, especiarias, doces) ${ }^{8}$, bebidas ácidas ou escovação frequente após vômitos ${ }^{29}$.

$\mathrm{O}$ esmalte dentário é influenciado pelo $\mathrm{pH}$ e está sujeito a contínuos processos de desmineralização/remineralização, mantendo a estrutura do meio bucal em equilíbrio com pH 7. A desmineralização do esmalte começa a partir de $\mathrm{pH}$ 5,5. Para os vômitos, um estudo identificou um valor médio de $\mathrm{pH} 3,8^{30}$, enquanto para bebidas de frequente consumo, o pH oscila entre 2,95 e 3,26, e iogurtes e leites aromatizados, entre 3,73 e 4,96. A ingestão frequente deste tipo de bebidas, comum em pacientes bulímicos, produz lesões erosivas em pacientes vulneráveis ${ }^{31}$.

O grau de erosão dental pode ser agravado por hábitos de higiene oral como a escovação de forma agressiva e repetitiva logo após a regurgitação. Neste momento o esmalte encontra-se desorganizado e pode ser removido facilmente pela abrasão durante a higiene oral ${ }^{12,22}$. Além disso, a inserção de dedos ou escovas de dente na cavidade oral pode causar pequenas lacerações no tecido da orofaringe. Estas injúrias podem ser exacerbadas se o conteúdo gástrico do estômago entrar em contato com essas áreas ${ }^{15}$.

Em estudo realizado para verificar os efeitos do vômito frequente na saúde dental em 35 pacientes bulímicas, os autores encontraram erosão dentária, abrasão e atrito, em 22 voluntárias da amostra, ou seja, 5-6 vezes mais frequente em bulímicos do que no grupo controle de 105 voluntários. Em relação à frequência dos vômitos, 25 dos 35 bulímicos vomitaram diariamente, 8 semanalmente e 2 nunca ${ }^{32}$. Pensando na dinâmica da deglutição, pode-se considerar o alto grau de possibilidade desses indivíduos bulímicos apresentarem hiposensibilidade dos elementos dentários em virtude da erosão, além de alterações nas fases oral e faríngea, em virtude da exposição das estruturas do trânsito orofaríngeo ao mecanismo agressivo do ato de vomitar.

Já a incidência de cáries é um fato controverso. Alguns autores defendem que é um achado comum $4,7,8,10,12,19,20,22$, resultante de uma excessiva ingestão de carboidratos e açúcares e uma pobre higiene oral ${ }^{8,22}$. Medicamentos antidepressivos podem contribuir para esse aumento de cáries, pois provocam xerostomia nestes pacientes ${ }^{8}$, o que pode dificultar a emulsificação do bolo alimentar durante a fase oral da deglutição. Entretanto, outros autores referem que apesar do grande consumo de carboidratos e gordura, os pacientes bulímicos possuem índice de cáries variável como na população normal ${ }^{12,33}$. Além disso, outro estudo refere baixo índice de cáries, assim como de placas e doença periodontal, dado justificado pelos autores como sendo resultado das práticas de higiene oral compulsivas ${ }^{14}$.

A erosão dos tecidos duros do dente também produz hipersensibilidade, alterações da oclusão, enfraquecimento e fratura das peças dentárias ${ }^{12,34}$, sendo todos esses possíveis fatores predisponentes à disfagia orofaríngea. $\mathrm{O}$ ácido do estômago 
que destrói o esmalte do dente também pode irritar a mucosa oral. Existem poucos estudos que referem como o vômito frequente em bulímicos pode afetar os tecidos e terminações sensitivas nessas áreas ${ }^{15}$.

Um desses estudos refere que as queixas de sensibilidade dentária ao quente, frio ${ }^{10,15}$ e substâncias ácidas podem ser atribuídas ao dano sofrido pelo esmalte dentário. Contudo, os pacientes também se queixam de respostas sensoriais ao estímulo doloroso em outras partes do corpo. Mais estudos são necessários em relação à sensibilidade orofaríngea em bulímicos, pois as fases oral e faríngea da deglutição podem estar alteradas secundariamente ao reconhecimento de características do alimento como volume, viscosidade e temperatura ${ }^{15}$.

Ainda em relação à sensibilidade, a literatura menciona que indivíduos com uma longa história de bulimia tem um grande risco de desenvolver uma redução da sensibilidade gustativa (hipogeusia) 10,18,35. Não há explicação clara para este achado, pois os estudos realizados não dividem os bulímicos por método de compensação. Alguns autores especulam que a escolha de bulímicos por comidas doces pode refletir um efeito fisiológico de ânsia/ desejo por este tipo de comida ${ }^{15}$. Além disso, 0 contato periódico da região intra-oral com o vômito, pode ser um fator que contribua para a hipogeusia.

Outro dado encontrado em pacientes que vomitam frequentemente é a papila interdental geralmente aumentada em consequência da constante irritação provocada pelo ácido do vômito. O status gengival pode estar igualmente afetado, caracterizado por gengivite e ressecamento da mucosa gengival ${ }^{36}$, também influenciado por medicações psiquiátricas ${ }^{33}$. Foram identificadas, ainda, aparições de granuloma e úlceras a nível oral ${ }^{10}$. Sendo assim, pode-se encontrar nesses pacientes alterações importantes na deglutição relacionadas à sensação de dor.

O aumento da secreção de amilase e o crescimento da glândula parótida também são frequentemente verificados na bulimia. Este crescimento pode ocorrer uni ou bilateralmente, raramente é doloroso ${ }^{15,37}$ e tende a aumentar a consistência ao toque ${ }^{10}$. O aumento do tamanho das glândulas parótidas é observado entre $8 \%$ e $59 \%$ de pacientes e também pode afetar outras glândulas salivares. A glândula parótida hipertrofiada pode ser observada entre 2 a 6 dias após um episódio de compulsão alimentar ${ }^{10,38}$, sendo seu aparecimento e magnitude diretamente proporcional à frequência de vômitos. Normalmente, o edema é submaxilar e vem acompanhado de xerostomia, podendo ser desfigurante e emocionalmente perturbador para um paciente bulímico, que já tem um quadro de ansiedade e depressão característico 8,14,37.

Existe a hipótese de que o estímulo colinérgico associado aos vômitos ativa as glândulas parótidas, aumentando a sua dimensão ${ }^{39}$. Outros autores acreditam que as enzimas pancreáticas proteolíticas levadas à boca durante os vômitos estimulam os receptores linguais gustativos, produzindo um aumento do estímulo autônomo da glândula, produzindo a hipertrofia ${ }^{38,40}$. Apesar destas justificativas, a razão para a ocorrência desta hipertrofia ainda é pouco conhecida ${ }^{15}$. Seu envolvimento com a deglutição pode estar relacionado às alterações na produção de saliva como ocorre na xerostomia.

Recentemente, um estudo identificou em pacientes bulímicos, achados como região em volta dos lábios com presença de eritema, descamação, sulcos e atrofia da mucosa labial, cujo diagnóstico é queilite descamativa crônica ${ }^{41}$, caracterizada por palidez, maceração e nos casos mais graves fissuras dolorosas nos ângulos da boca ${ }^{14}$. A etiologia da queilite angular também está associada à presença do fungo Candida albicans ${ }^{35}$. Nota-se, portanto, que tais achados podem estar associados até mesmo às queixas na fase preparatória oral da deglutição.

Neste mesmo estudo, verificou-se que o palato mole apresenta coloração amarelada intensa relacionada com a alta ingestão de carotenos. Outro importante achado consiste na presença de hematomas na úvula ${ }^{41}$, resultado do ato agressivo de indução do vômito com o dedo, além de sua exposição ao meio ácido.

Algumas características clínicas discutidas até aqui foram observadas em pesquisa realizada com uma amostra de 70 pacientes com transtornos alimentares: sinal de Russell, hipertrofia de glândula salivares e erosão dentária de gravidade importante, devido à perda irreversível dos tecidos dentários. Além disso, os pacientes que apresentaram o hábito de vomitar foram mais susceptíveis às cáries e a prevalência de hipertrofia de glândulas salivares foi encontrada em $9,52 \%$ da amostra. As causas não foram firmemente estabelecidas, entretanto foi associada aos episódios de vômitos ${ }^{42}$.

\section{Faringe}

Assim como a cavidade oral, a região faríngea pode manifestar modificações morfofuncionais decorrentes de seu contato sistemático com o meio ácido do vômito em pacientes bulímicos.

Alguns sinais e sintomas podem ser observados, como presença de tosse, odinofagia e ulceração em região faríngea. Todos são indícios de alterações no trânsito alimentar, constituindo eventos característicos da disfagia. 
Anormalidades na deglutição podem se tornar um problema na população bulímica se aspectos do sistema sensorial estiverem sendo alterados pelo estímulo persistente do reflexo e episódios de vômitos ou se existirem outras alterações estruturais decorrentes do ato de vomitar. Quando o bulímico estimula mecanicamente o vômito, ele também está interferindo potencialmente com o sistema sensorial e mecanorreceptores nesta região, o que pode influenciar em aspectos da deglutição ${ }^{15}$.

As complicações mais comuns na região faríngea de bulímicos são dor ao engolir, vômitos com sangue e inflamação na faringe pelos efeitos do vômito ${ }^{15,37}$. A dor de garganta crônica pode ser devido à irritação esofágica causada tanto pelo vômito quanto pelo refluxo gastroesofágico ${ }^{29}$.

A estimulação mecânica sistemática do reflexo de vômito pode dessensibilizar a região ${ }^{15,43}$. A redução da sensibilidade em orofaringe leva o indivíduo bulímico a tentar eliciar o reflexo de vômito mais posteriormente, na faringe, o que o leva a utilizar objetos longos, como a escova de dentes para alcançar a região ${ }^{15}$.

\section{Deglutição e Disfagia}

Poucos estudos com a população bulímica têm como foco principal as possíveis dificuldades de deglutição deste grupo. Como foi relatado anteriormente, indivíduos com BN têm risco potencial de desenvolver disfagia orofaríngea, dada às alterações morfofuncionais provocadas pela regurgitação ácida do conteúdo gástrico.

$\mathrm{O}$ primeiro relato de disfagia relacionada à $\mathrm{BN}$ na literatura foi de uma mulher caucasiana de 40 anos ${ }^{44}$. Grande volume de alimento passou a ficar alojado em região esternal e a BN foi considerada a causa. Diante deste caso, os autores sugerem que a disfagia deve ser sempre investigada como um distúrbio básico na BN.

$\mathrm{Na}$ literatura consultada apenas um estudo descreveu os padrões da deglutição orofaríngea em sujeitos bulímicos. Neste trabalho, os autores realizaram exame de videofluoroscopia em pacientes com história de BN. A partir dos resultados do método, eles concluíram que pacientes bulímicos possuem dois tipos característicos, aos quais eles chamaram de $A$ e $B$. No tipo $A{ }^{15}$, observaram-se contrações da orofaringe após a total passagem do bolo alimentar pela faringe e entrada no esôfago. Tais contrações iniciaram na hipofaringe e se moveram superiormente através da laringe, epiglote, base de língua e véu palatino. $O$ tipo $B$ mostra deficiência no transporte do bolo alimentar pela faringe, resultado da diminuição de força da língua, provocando acúmulo de resíduo em região faríngea ${ }^{43}$.
A hipótese dos autores para justificar os resultados concentra-se na força insuficiente da língua para realizar adequadamente seu movimento durante a deglutição. No entanto, outros autores levantam uma diferente hipótese, como a possibilidade de redução da sensibilidade faríngea pelos frequentes episódios de vômitos, o que fisiologicamente parece ser mais coerente com o quadro apresentado na BN. Além disso, manifestam críticas a esse estudo e mencionam que nele não há grupo controle, não fica claro o tipo de informação retirada do exame videofluoroscópico e não há informações sobre o tipo e quantidade de alimento ofertado ${ }^{15}$.

Outra pesquisa hipotetizou que pacientes com disfagia psicogênica, cujo diagnóstico é incerto, podem ter algum tipo de TA, tais como anorexia nervosa ou bulimia nervosa. Tanto a disfagia quanto $o$ TA possuem características similares, como queda do apetite, ingestão reduzida de comida, seleção de alimentos, alterações sensoriais orais, mastigação e atividades relacionadas à preparação do bolo alimentar. Por meio da aplicação de protocolos, o estudo demonstrou que 21 pacientes com disfagia psicogênica parecem não ter nenhum tipo de TA, porém os resultados demonstram níveis significantes de estresse, especialmente ansiedade, dificuldade de relação interpessoal e depressão, fatores que podem servir como indicadores no diagnóstico diferencial de disfagia psicogênica ${ }^{45}$.

Em uma pesquisa realizada recentemente, buscou-se determinar quais alterações nas funções gastrointestinais são mais frequentes em 101 sujeitos com transtornos alimentares, incluindo BN (22\% da amostra). A disfagia ficou em $5^{\circ}$ lugar dentre as encontradas $(23 \%)^{46}$, porém neste caso, não ficou claro a que tipo de disfagia o estudo se refere (orofaríngea ou esofágica), que fase da deglutição está afetada, nem quais os critérios utilizados para que o indivíduo fosse considerado disfágico.

Na literatura, é possível encontrar que os danos aos receptores sensoriais ao nível da faringe poderiam provocar um retardo no mecanismo da deglutição, e consequentemente, favorecer a passagem de alimentos para via aérea. Além disso, as microaspirações que acompanham os vômitos podem resultar em bronco-espasmos, asma e pneumonia ${ }^{10}$.

A partir do que foi descrito anteriormente, podese afiançar que, as consequências físicas da BN são bem estabelecidas na literatura, no entanto, 0 impacto funcional é menos claro. Isso foi constatado em trabalho de revisão ${ }^{15}$ realizado no início da década atual. Neste trabalho, os autores realizaram uma revisão crítica sobre as alterações que podem gerar problemas de deglutição em pacientes bulímicos, observaram a escassez de trabalhos sobre a relação entre a bulimia e esta função, e 
alertaram sobre a importância dos profissionais de saúde estarem atentos a isso. Ainda assim, após esse estudo, pouco se encontrou na literatura sobre esta relação.

Pelo envolvimento da BN com as estruturas e funções que compõem o sistema sensório-motor oral, pode-se considerar que o fonoaudiólogo pode se inserir clínica e cientificamente nessa área, uma vez que possui propriedade para tal. Apesar desta constatação óbvia, não foi encontrada na literatura nenhuma referência que mencionasse isso claramente, assim como não se observam estudos realizados por fonoaudiólogos com esta população.

\section{CONCLUSÃO}

Nota-se por meio dessa revisão de literatura que a bulimia nervosa acarreta uma série de alte- rações em estruturas e funções que compõem o trânsito orofaríngeo, tais como erosão dentária, hipersensibilidade, enfraquecimento e fratura dos dentes, problemas de oclusão, cáries, doenças periodontais, dessensibilização inra-oral, hipogeusia, úlceras, granulomas, queilite angular, hipertrofia das glândulas parótidas, tosse e odinofagia. Percebe-se que existe uma abordagem bem mais frequente de sinais, sintomas e seqüelas morfológicas em detrimento das funcionais, existindo um número reduzido de pesquisas que mencionam o envolvimento entre a bulimia e alterações na deglutição.

Sendo esta função objeto de estudo da Fonoaudiologia, faz-se necessário que sejam realizadas mais pesquisas metodologicamente bem estruturadas na área, considerando a existência ainda restrita de conhecimento sobre o tema.

\begin{abstract}
Background: deglutition and bulimia. Purpose: to submit and discuss the scientific research concerning oropharyngeal characteristics related to deglutition in patients with purging type bulimia nervosa. Conclusions: bulimia nervosa entails a series of changes in structures and functions that compose the oropharyngeal tract, such as dental erosion, hypersensitivity, weakness and fracture of the teeth, occlusion problems, caries, periodontal diseases, intraoral desensitization, hypogeusia, ulceration, granulomas, angular cheilitis, enlargement of the parotid glands, coughs and odynophagia. There is a prevalence in the scientific literature of reports about morphologic alterations on the detriment of the functional ones. Few reports discussed the relationship between bulimia and deglutition, superficially mentioning the possibilities for triggering oropharyngeal dysphagia.
\end{abstract}

KEYWORDS: Eating Disorders; Bulimia; Deglutition

\section{REFERÊNCIAS}

1. Oliveira EA, Santos MA. Perfil psicológico de pacientes com anorexia e bulimia nervosas: a ótica do psicodiagnóstico. Medicina. 2006; 39(3):353-60.

2. Cabrera CC. Estratégias de intervenção interdisciplinar no cuidado com o paciente com transtorno alimentar: o tratamento farmacológico. Medicina. 2006; 39(3):375-80.

3. Fernandes CAM, Rodrigues AP, Nozaki VT, Marcon SS. Fatores de risco para o desenvolvimento de transtornos alimentares: um estudo em universitárias de uma instituição de ensino particular. Arq Ciênc Saúde Unipar. 2007; 11(1):33-8.

4. Borges NJBG, Sicchieri JMF, Ribeiro RPP, Marchini JS, Santos JE. Transtornos alimentares: quadro clínico. Medicina. 2006; 39(3):340-8.
5. Puentes MS. La bulimia nerviosa y sus subtipos. Diversitas Perspectiv Psicol. 2005; 1(1):79-87.

6. Espíndola CR, Blay SL. Bulimia e transtorno da compulsão alimentar periódica: revisão sistemática e metassíntese. Rev Psiquiatr. 2006; 28(3):265-75.

7. Bonsignore AM. Bulimia nerviosa y otros trastornos de la conducta alimentaria: algunas reflexiones. Arch Argent Pediatr. 2004; 102(6):419-21.

8. Zárate OG, Rodríguez GE. Anorexia y bulimia nerviosa. Aspectos odontológicos. Rev ADM. 2000; 57(1):23-32.

9. Kaye W. Neurobiology of anorexia and bulimia nervosa. Physiol Behav. 2008; 94(1):121-35.

10. Rava FM, Silber TJ. Bulimia nerviosa (parte 1): historia, definición, epidemiología, cuadro clínico y complicaciones. Arch Argent Pediatr. 2004; 102(5):353-63. 
11. Paccola ATF. Escuta do psiquiatra: sinais e sintomas de anorexia nervosa e bulimia nervosa. Medicina. 2006; 39(3):349-52.

12. Traebert J, Moreira EAM. Transtornos alimentares de ordem comportamental e seus efeitos sobre a saúde bucal na adolescência. Pesq Odontol Bras. 2001; 15(4):359-63.

13. Winstead NS, Willard SG. Gatrointestinal complaints in patients with eating disorders. J Clin Gastroenterol. 2006; 40(8):678-82.

14. Mehler PS, Crews $C$, Weiner K. Bulimia: medical complications. J Womens Health (Larchmt). 2004; 13(6):668-75.

15. Mendell DA, Logemann JA. Bulimia and swallowing: cause for concern. Int $\mathrm{J}$ Eating Disord. 2001; 30(3):252-8.

16. Silva LM, Santos MA. Construindo pontes: relato de experiência de uma equipe multidisciplinar em transtornos alimentares. Medicina. 2006; 39(3):415-24.

17. Russell G. Bulimia nervosa: an ominous variant of anorexia nervosa. Psychol Med. 1979; 9(3):429-48.

18. Anderson L, Shaw JM, McCargar L. Physiological effects of bulimia nervosa on the gastrointestinal tract. Can J Gastroenterol. 1997; 11(5):451-9.

19. Caldeira TH, Ottaviano-Nápole RC, Busse SR. Bulimia nervosa e suas alterações odontológicas. Pediatr Mod. 1999; 35(5):288-96.

20. Maso AA, Ayala MC, Rivas ZG, Mora T. Bulimia: revisión bibliográfica. Acta Odontol Venezol. 2001; 39(2):70-3.

21. Sicchieri JMF, Bighetti F, Borges NJBG, Santos E, Ribeiro RPP. Manejo nutricional nos transtornos alimentares. Medicina. 2006; 39(3):371-4.

22. Seabra BGM, Seabra FRG, Almeida RQ, Ferreira JMS. Anorexia nervosa e bulimia nervosa e seus efeitos sobre a saúde bucal. Rev Bras Patol Oral. 2004; 3(4):195-8.

23. Alvarenga $M$, Larino MA. Terapia nutricional na anorexia e bulimia nervosa. Rev Bras Psiquiatr. 2002; 24(Supl3):39-43.

24. Bacaltchuk J, Hay P. Tratamento da bulimia nervosa: síntese das evidências. Rev Bras Psiquiatr. 1999; 21(3):184-7.

25. Mello Neto GAR, Martinez VCV, Santos FO, Silva Junior MC. Anorexia e bulimia, suas interfaces com a histeria e o discurso psicanalítico. Aletheia. 2006; (23):101-11.

26. Hellström I. Oral complications in anorexia nervosa. Scand J Dent Res. 1977; 85(1):71-86.

27. Bouquot JE, Seime RJ. Bulimia nervosa. Dental perspectives. Pract Perio Aesth Dent. 1997; 9(6):655-64.

28. National Eating Disorders Association. Dental complications of eating disorders: information for dental practitioners. [homepage na internet] Seattle: 2002. [acesso em 2 maio 2006] Disponível em: http://www.edap.org/p. asp?WebPage_ID=286\&Profile_ID=73512

29. Uyeda L, Tyler I, Pinzon J, Birmingham CL. Identification of patients with eating disorders. The signs and symptoms of anorexia nervosa and bulimia nervosa. Eat Weight Dis. 2002; 7(2):116-23. 30. Milosevic A, Brodie DA, Slade PD. Dental erosion, oral hygiene and nutrition in eating disorders. Int $\mathrm{J}$ Eat Disord. 1997; 21(2):195-9.

31. Argentieri A, Pistochini A, Doño R, Añón MC, Tognaccioli L. Determinaciones analíticas de bebidas no alcohólicas. Bol Asoc Argent Odontol Niños. 2002; 31(4):9-14.

32. Rytomaa I, Jarvinen V, Kanerva R, Heinonen OP. Bulimia and thooth erosion. Acta Odontol Scand. 1998; 56(1):36-40.

33. Kavoura V, Kourtis SG, Zoidis P, Andritsakis DP, Doukodakis A. Full-mouth rehabilitation of a patient with bulimia nervosa. A case report. Quint Int. 2005; 36(7):501-10.

34. Burke FJ, Bell TJ, Ismail N, Hartley P. Bulimia: implications for the practising dentist. Brit Dent J. 1996; 180(11):421-6.

35. Frydrych AM, Davies GR, McDermott BM. Eating disorders and oral health: a review of the literature. Aust Dental J. 2005; 50(1):6-15.

36. Alonso C, Sabás M, Castillo MA, Weisstaub G, Pascual DM. Transtornos de la conducta alimentaria: repercusiones a nivel oral. Rev Assoc Odontol Argent. 2001 Jul-Ago; 89(4):390-5.

37. Ballone GJ. Bulimia nervosa. Rev Psiquiatr. 2003; 21:45-7.

38. Wittich GR, Scheible WF, Hajek PC. Ultrasonography of the salivary glands. Radiol Clin North Am. 1985; 23(1):29-37.

39. Rauch SD, Herzog DB. Parotidectomy for bulimia: a dissenting view. Am J Otolaryngol. 1987; 8(6):376-80.

40. Ogren FP, Huerter JV, Pearson PH, Antonson $\mathrm{CW}$, Moore GF. Transient salivary gland hypertrophy in bulimics. Laryngoscope. 1987; 97(8):951-3.

41. Panico RL. Sintomas y signos bucales en pacientes con bulimia y anorexia nerviosa. Rev Fac Cienc Med Córdoba. 2006; 63(Suppl2):30-2.

42. Berner E, Piñero J, Valente S. Trastornos de la conducta alimentaria: signos clínicos en pacientes adolescentes con episodios de vómitos autoinducidos. Arch Argent Pediatr. 2004; 102(6):440-4.

43. Roberts MW, Tylenda CA, Sonies BC, Elin RJ. Dysphagia in bulimia nervosa. Dysphagia. 1989; 4(2):106-11. 
44. Birmingham CL, Chang PC, Cleator LGM. Mechanical dysphagia in a patient with bulimia. Int $\mathrm{J}$ Eat Disord. 1987; 6(4):561-4.

45. Barofsky I, Fontaine KR. Do psychogenic dysphagia patients have an eating disorder? Dysphagia. 1998; 13(1):24-7.
46. Boyd C, Abraham S, Kellow J. Psychological features are important predictors of functional gastrointestinal disorders in patients with eating disorders. Scand J Gastroenterol. 2005; 40(8):929-35.

RECEBIDO EM: 04/01/2009

ACEITO EM: 19/10/2009

Endereço para correspondência:

Leandro de Araújo Pernambuco

Hospital de Câncer de Pernambuco -

Serviço de Fonoaudiologia

Av. Cruz Cabuga, 1597

Recife - PE

CEP: $50040-000$

Email: leandroape@globo.com

Rev. CEFAC. 2010 Mar-Abr; 12(2):308-316 